\title{
Driver and Passenger Identification from Smartphone Data
}

\author{
Bashar I. Ahmad, Patrick M. Langdon, Jiaming Liang, Simon J. Godsill, Mauricio Delgado and Thomas Popham
}

\begin{abstract}
The objective of this paper is twofold. First, it presents a brief overview of existing driver and passenger identification or recognition approaches which rely on smartphone data. This includes listing the typically available sensory measurements and highlighting a few key practical considerations for automotive settings. Second, a simple identification method that utilises the smartphone inertial measurements and, possibly, doors signal is proposed. It is based on analysing the user behaviour during entry, namely the direction of turning, and extracting relevant salient features, which are distinctive depending on the side of entry to the vehicle. This is followed by applying a suitable classifier and decision criterion. Experimental data is shown to demonstrate the usefulness and effectiveness of the introduced probabilistic, low-complexity, identification technique.
\end{abstract}

Index Terms-Identification, connected vehicles, classification, intelligent vehicles, sensor data fusion.

\section{INTRODUCTION}

\section{A. Background and Motivation}

There has been lately a considerable interest in leveraging the recent advances in the sensing, data storage-processing and wireless communications technologies in vehicles to introduce smart functionalities. Their aim is to offer drivers and passengers, not only safer, but also a personalised and more pleasant driving experience [1], [2]. This goes beyond the classical Advanced Driver Assistance Systems (ADAS) [3], [4] and route guidance services [5] to customising the vehicle interior and adapting its systems to the driver and passenger(s) profiles and preferences [6], for example seat positions, setting reminders, temperature control, HMI, infotainment system, etc. Nevertheless, such functionalities rely fundamentally on identifying the vehicle user [7], [8], particularly when a vehicle has multiple drivers. Most importantly, they require labelled pertinent data, i.e. for a known user, from various sources such as in-vehicle sensing systems or smartphones or even infrastructure, to learn preferences, profiles and behaviours.

Driver identification is also relevant to insurance telematics, for instance the driving style can guide setting the user's premium by insurance firms [9]. Establishing this style can be based on recorded data from the vehicle On-Board Diagnostics (OBD) system or present smartphone(s), assuming a known driver identity. Other automotive applications that require data tagging, thereby driver recognition, encompass those aimed at reducing the carbon footprint of driving as per the user's travel history, traffic status and others [10].

B. I. Ahmad, P. M. Langdon, J. Liang and S. J. Godsill are with the Engineering Department, University of Cambridge, Trumpington Street, Cambridge, UK, CB2 1PZ. Emails: \{bia23, pml24, j1809, sjg30\}@cam.ac.uk.

M. Delgado and T. Popham are with Jaguar Land Rover (JLR), Coventry, UK. Emails: \{tpopham, amunozd1\}@jaguarlandrover.com.
On the other hand, the remarkably fast growth of smartphone ownership has motivated the move towards exploiting smartphones versatile set of sensors, such as the Global Navigation Satellite System (GNSS) receiver and Inertial Measurement Units (IMUs), in automotive applications. Examples include: traffic state estimation [11], navigation [12], driver assistance [13] and many others. Interestingly, the problem of determining the smartphone to vehicle position is closely related (or corresponds) to the driver and passenger(s) identification task. This capitalises on the premise that the smartphone is:

1) usually in the vicinity of its owner, and

2) a personal item, which is not shared with other users, unlike a (smart) key-fob, which can be used/shared by multiple vehicle drivers.

Smartphone-to-vehicle localisation, which covers inside and/or outside the vehicle, hence enables identifying the present vehicle user(s), i.e. if the smartphone owner is the driver or, front or rear passenger. Recognition can be performed before or after entering the car. Locating the phone within the vehicle can, amongst others, be employed to minimise distractions induced by using a smartphone whilst driving. For example, the driver's smartphone services and functionalities can be accordingly restricted [9], [14].

Additionally, realising a connected cooperative vehicle environment is currently attracting immense interest from researchers and OEMs around the world, mainly due to its importance to autonomous driving [15]-[17]. This includes vehicle to vehicle, vehicle to infrastructure and vehicle to cloud communications, typically with stringent latency and performance requirements. Thus, a smartphone user identification solution can exchange data with the vehicle in a connected set-up. It can also have access to the vehicle data (e.g. doors signal, which indicates whether a given vehicle door is opened or closed), user's calendar, journeys history, etc.

Therefore, a smartphone-based driver/passenger(s) identification or phone-to-vehicle localisation, possibly in a connected vehicle environment, has various applications in intelligent vehicles. This comprises, but not limited to, delivering personalised driving experience via adapting in-vehicle systems, insurance telematics and minimising distractions. Vehicle keyless entry systems, authentication and security in general, are other areas that can benefit from an additional modality for confirming the identity of the present user(s), i.e. from his/her smartphone data [18], [19]. In this paper, various categories of existing smartphone-based driver and passenger(s) recognition techniques are outlined. A simple, novel, identification approach is subsequently proposed and evaluated. 


\section{B. Contributions}

The contributions of this paper are twofold. First, it gives a brief overview of the main six categories of non-intrusive smartphone-based identification approaches. This excludes those that involve the drivers/passengers actively identifying themselves to the vehicle, e.g. via a specialised automotive hardware-firmware for face or voice recognition. The key features and limitations of the addressed techniques are outlined. This follows listing relevant smartphone sensory data and highlighting a few crucial practical considerations.

Second, a low-complexity driver/passenger(s) identification method is introduced. It fuses the smartphone sensory data and can utilise vehicle signals (namely, doors signal) in a connected environment. The proposed approach relies on analysing the user motion during entry to vehicle, capturing well defined salient features that vary depending on the entry side (driver or passenger). It considers the gradient of the turning angle during the entry micro-movements. Extracted features are utilised by a classifier to determine the probability of the user being a driver or passenger. A decision criterion is then applied; it can employ the doors signal to distinguish between front or rear present vehicle users. Unlike comparable prior work, e.g. in [14], [20], the smartphonebased method introduced here is: a) independent of the phone position and orientation (thereby does not impose restrictions on where the user has to carry the phone during-after entry), b) unsusceptible to errors induced by, potentially, low quality smartphone sensory data, and c) less sensitive to variations in the entry behaviour. Overall, this paper presents a simple, yet effective and generic, novel probabilistic smartphone driver/passenger(s) recognition approach. It is enabled by a principled treatment of the considered classification problem, careful processing of the sensory data and clearly identifying representative features.

Finally, experimental data from various pilot studies and under several conditions illustrate the efficacy of the proposed driver/passenger recognition technique, with and without the availability of the doors signal.

\section{Paper Outline}

The remainder of this paper is organised as follows. In Section II, smartphone sensory data and various considerations are listed. A short overview of existing identification methods, including those reliant on analysing the user behaviour, is given in Section III. The proposed identification approach is described in Section IV and its performance is assessed in Section V. Conclusions are drawn in Section VI.

\section{Sensory Data And Practical Considerations}

The common sensors/receivers in a smartphone, particularly those relevant to the phone-to-vehicle positioning (identification) problem, can be categorised into (see Table I):

- Inertial sensors: accelerometer(s) and gyroscope(s).

- Exteroceptive sensors/receivers : GNSS, magnetometer, camera(s), microphone(s), Bluetooth/BLE, WiFi, cellular and Near Field Communication (NFC) and others.

In a connected setup (e.g. via a mobile or local network) smartphones can additionally receive, with minimum delay
[16], vehicle data and user information. The former can incorporate the (raw or processed) signals: doors, suspensions, steering, braking, pedals press and others. Such data can be received from custom-made in-vehicle loggers or directly from the OBD via a dongle [21]. Whereas, other user information can be available via the cloud, e.g. calendar, travel history, etc.

Despite this wide range of phone sensory data, an identification solution should consider the following practical issues:

- Data quality: built-in smartphone sensors are not primarily designed for vehicle telematics or accurate localisation applications. For example, a smartphone IMU chipset can belong to a low grade of inertial sensors since their use is typically limited to correctly adjusting the phone display orientation (portrait or landscape). Whilst these IMUs can provide data rates in the range of $20-300 \mathrm{~Hz}$, their data can exhibit significant bias, drift factor, misalignment, and random errors [22]. However, employing suitable data fusion and statistical filtering algorithms can mitigate the impact of such measurements imprecisions [23], [24].

- Phone Position: the phone can be held in arbitrary positions-orientations (e.g. hand, pocket and bag), which cannot be assumed to be fixed during approaching or entering the vehicle. This causes ambiguities to processing data from orientation dependent sensors such as IMUs.

- Automotive Settings: indoor (e.g. underground or covered car parks) and/or dense urban environments can have detrimental impact on the data quality from several smartphone exteroceptive receivers due to occlusions and multipath fading in complex settings. For example, quality of GNSS data (if available) can be poor [24]. Similarly, the coverage or Received Signal Strength Indicator (RSSI) for RF transmissions, e.g. Bluetooth/BLE, WiFi and cellular, can be notably attenuated, especially when there are obstacles (cars and walls) between the phone and transmitters. Contrary to classical indoor positioning applications, here obstacles and environment can dynamically change, e.g. parked cars, pedestrians, etc.

- Power Consumption: the power consumption of data collection and processing of any smartphone-based service is critical, given the limited available power resources. For example, IMU sensors utilised in the identification

TABLE I: Selected smartphone sensors/receivers relevant to the smartphone-to-vehicle positioning problem and their measurements.

\begin{tabular}{|c|c|}
\hline Sensor/Receiver & Observations \\
\hline \multicolumn{2}{|r|}{ Inertial } \\
\hline Accelerometer & Acceleration across $\mathrm{x}, \mathrm{y}$ and $\mathrm{z}$ axis. \\
\hline Gyroscope & Angular velocity across $\mathrm{x}, \mathrm{y}$ and $\mathrm{z}$ axis. \\
\hline \multicolumn{2}{|r|}{ Exteroceptive } \\
\hline GNSS & Position, planar speed/course, etc. \\
\hline Magnetometers & Magnetic flux across $\mathrm{x}, \mathrm{y}$ and $\mathrm{z}$ axis. \\
\hline Barometer & Atmospheric pressure (altitude data). \\
\hline Bluetooth/BLE & RSSI and source/recipient credentials ${ }^{\dagger}$. \\
\hline NFC & Source/recipient credentials ${ }^{\dagger}$ \\
\hline WiFi and Cellular & RSSI and source credentials ${ }^{\dagger}$. \\
\hline Cameras & Images. \\
\hline Microphones & Audio. \\
\hline \multicolumn{2}{|c|}{$\dagger$ Data can also be exchanged with source/recipient or via a network. } \\
\hline
\end{tabular}


method proposed in this paper, consume, on average, substantially less power compared with GNSS [25]; even with sampling the GNSS receiver at low rates due to its cold/warm/hot-start nature [9], [26].

In the next section, we briefly describe key identification techniques that rely on smartphone sensory measurements.

\section{RELATED WORK ON SMARTPHONE-BASED IDENTIFICATION: AN OVERVIEW}

Figure 1 depicts the in-vehicle regions for the Driver (D), Passenger Front (P-F), Passenger Rear Offside (P-RO) and Passenger Rear Nearside (P-RN), in the UK; these regions can be analogously defined for a left hand drive configuration. The identification (smartphone-to-vehicle positioning) solution aims to associate a present user/smartphone with one of these four classes. In general, smartphone-based driver/passenger(s) identification approaches can be divided into six categories. They are listed in Table II, highlighting when and where the classification is accomplished. Certain methods can only identify a subset of the potential four users in Fig. 1, see Table II. We recall that intrusive none-smartphone-based techniques, which can involve the users identifying themselves to the vehicle (e.g. via a biometric scanner or an HMI interface for ID entry or camera for face recognition and others) are not discussed here.

\section{A. Accurate Localisation}

The ubiquitous GNSS positioning services on smartphones is the obvious candidate for providing the sought phone-tovehicle localisation information, including the user's exact seat position, i.e. D, P-F, P-RO and P-RN. However, differentiating between a driver and a passenger demands an accuracy higher than, approximately, half the width or length of the vehicle, e.g. errors should be less than $0.75 \mathrm{~m}$. This performance requirement is excessively onerous for a smartphone localisation service [24], especially in indoor/covered car parks or dense urban areas. This is in addition to the high power consumption of GNSS solutions, quickly draining the phone battery [25].

Nonetheless, the current advances in Pedestrian Dead Reckoning (PDR) and indoor positioning can enable smartphoneto-vehicle positioning that meets high accuracy specifications

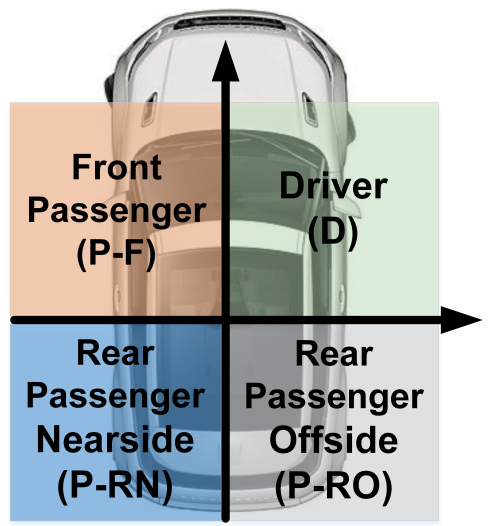

Fig. 1: Driver and passengers nonoverlapping zones in or near the vehicle; they can be accordingly extended. (e.g. less than $50 \mathrm{~cm}$ ) by fusing various sensory data such as inertial data, magnetometer, RSSI (either opportunistically, e.g. from existing WiFi and cellular transmitters, or from dedicated on-vehicle RF transmitters, e.g. bluetooth beacons) and possibly an intermittent GNSS signal [24], [27]-[30]. Besides, ultrawideband (UWB) technology, which is poised to proliferate in smartphones, can deliver high accuracy positioning, e.g. by using on-vehicle UWB beacons to measure time-of-flight to smartphone [31], [32]. It is noted that proximity technologies are addressed separately in Section III-F.

\section{B. Analysing Human Motion}

Another common approach is to analyse the user motion during-after entering the vehicle from the phone sensory data; thus this category encompasses the driver/passenger(s) recognition technique introduced in this paper. As well as entry motion, other movements, such as those associated with seat-belt fastening and pedal pressing, can be considered [14], [20]. For instance, the phone IMU data related to entering the vehicle and seat-belt fastening incorporate distinct features depending on the entry side. Whereas, detecting a pedal press from the smartphone accelerometer data indicates that the user is the driver. This generally allows distinguishing between a driver and passenger, which suffices in certain applications such as tagging the driver data. It cannot however differentiate between front or rear passengers, e.g. the entry behaviours of both D and P-RO, who are both on the same vehicle side, can be indistinguishable from one another.

Analysing human motion during and after entering the vehicle can be susceptible to behavioural variations and errors due to the poor quality of the available sensory measurements such as IMU. Ambiguities in orientation dependent data (e.g. IMU), which can originate from the phone arbitrary positions, can also impose stringent restrictions on the phone location when driver/passenger approaches and enters the car. For instance, the turning motion and pedal pressing detectors in [14], [20] assume that the smartphone is in a lower body pocket. Seat-belt-related activity is detectable only when the phone is in a user's upper-body pocket [14]. Nevertheless and as proposed in Section IV, capturing representative features and using additional data leads to formulating a reliable and robust identification solution.

\section{Modelling Driving Behaviour}

Several studies have been conducted on performing driver identification by studying individual driving styles and travel behaviours [33], see [34] for an overview. This can include the pedal use, steering, braking, travel history, previous routes and even physiological signals (e.g. ECG and skin conductance). These methods typically have high training requirements (e.g. demand the availability of extensive labelled data sets) and entails considerable computational cost. Furthermore, detection is restricted to the driver, and it is accomplished after the start of the journey. Whilst signals such as speed and steering-turning can be obtained (albeit noisy) from the phone sensors, such solutions assume that certain OBD or wearable technology [35] data is available to the smartphone in a connected environment. 
TABLE II: Selected driver-passenger recognition approaches highlighting when, where and who can be identified.

\begin{tabular}{|c||c|c|c|}
\hline \hline Approach & Identification Time & Detect Inside/Outside Vehicle & Identifiable Users \\
\hline Accurate Localisation & Before and after entry to the vehicle & Inside and/or outside & All \\
\hline Motion Behaviour & During-after entry; before journey starts & Inside & Driver and passenger \\
\hline Driving Behaviour & After the start of the journey & Inside & Driver \\
\hline In-vehicle Forces & After the start of the journey & Inside & All \\
\hline Audio Ranging & After entry and/or during the journey & Inside & All \\
\hline Proximity/MF/Other & Before and after entry & Inside and/or outside & - \\
\hline \hline
\end{tabular}

\section{Forces within a Moving Vehicle}

This builds on the observation that specific forces, measured by accelerometers, vary depending on where in the vehicle the accelerometer is placed [36]-[38]. This is detectable during significant dynamical events, e.g. when passing a pothole or during notable cornering (lateral accelerations). Related methods require the presence of at least two collaborating invehicle IMUs located at different positions in the car interior, for instance a smartphone and a vehicle-fixed IMU. Since the difference in the specific forces reported by the two (or more) IMUs only depends on their relative positions, and not on their absolute locations, this identification solution is applicable when the "absolute" accurate position of one of the IMU sensors is known. The delay in accomplishing the user recognition task can be substantial, e.g. after the start of a journey. This relies on the presence or undertaking highly dynamical events; see [38] for a recent overview.

\section{E. Audio Ranging}

It employs the smartphone embedded microphone(s) to establish the phone (owner) in-vehicle position. It uses audio signals, of inaudible frequencies, emitted from several speakers of the vehicle stereo system, possibly in a programmed sequence [39], [40]. Such signals can be instigated by the smartphone, e.g. after it pairs with the vehicle infotainment system via Bluetooth. The generated audio signals are then recorded by the phone, and analysed to deduce the timing differentials, e.g. between the left, right, front and back speakers. From these differentials, the phone can self-determine its position in the vehicle. Hence, it triangulates its location to one of the four vehicle quadrants in Fig. 1. Other similar, basic, techniques measure the magnitude of emitted inaudible beeps by a given speaker [14], [20] or the vehicles turn signal [14], to establish the phone proximity to the audio signal source. In [40], a related seamless-voice-recognition approach is proposed.

Whilst audio-ranging-based user identification systems are in general accurate and robust [39], [40], they necessitate changing the vehicle firmware and recording audio in an unsupervised manner, which can raise privacy concerns.

\section{F. Dedicated Hardware, Proximity, Magnetic Field and Others}

Vehicle-installed RF technology can identify the driver or passenger from his/her smartphone; this might entail estimating the phone proximity to a given transceiver [41]. Examples include Bluetooth via paring (paring can result in a significant delay and only reveals the smartphone presence, not its invehicle position), Bluetooth Low Energy (BLE) beacons and NFC. The latter is of particular interest since modern vehicles can be equipped with NFC or RFID radio(s) for key-less entry or engine-start. NFC transceiver(s) can not only expedite the vehicle-phone pairing, they can identify the driver's smartphone (owner) or that closest to it, i.e. proximity. However, the NFC coverage is notably short, which reduces the classification range, and recognition can be limited to driver (e.g. when unlocking door). Other proximity sensors with longer ranges can be employed, e.g. BLE beacons in/on the vehicle [27], [30], [42], [43]. Nevertheless, any proximity-based solutions have to take into account the prevalent occlusions in the considered automotive scenarios.

Other signals measurable by the smartphone, such as Magnetic Field (MF) fluctuations due to starting the vehicle engine, can permit determining if the phone is in the front or back half of the vehicle as in [20]. Alternatively, the phone-to-vehicle position can be estimated, however coarse, by analysing the smartphone magnetometer observations [44]. Finally, amongst others, pressure sensors can be used [45], e.g. mounted on each seat to estimate the driver/passenger(s) weight(s). Prior knowledge of the users' weights can then facilitate identifying them by sharing this data with the smartphone.

Whilst the above brief overview serves the purpose of outlining the main visible smartphone-based driver/passenger(s) recognition techniques, several methods can be implemented by an identification system as in [14] and [20].

\section{Proposed Identification Approach}

The simple driver/passenger(s) identification approach introduced here predominately relies on analysing the user behaviour whilst entering the vehicle, namely his/her turning angle and direction. In particular, a driver has to turn clockwise during entry, whereas a front or rear-nearside passenger has to turn anticlockwise as depicted in Fig. 2. The proposed tech-

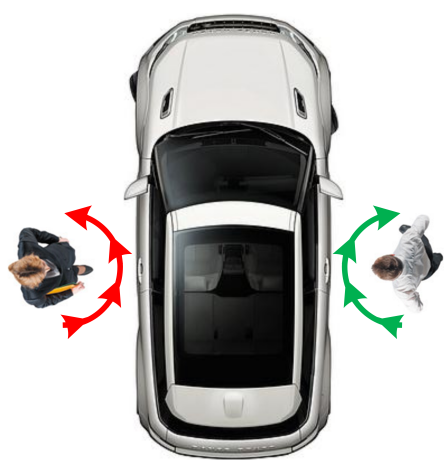

Fig. 2: Driver (right) and passenger (left); arrows show direction of turning during entry (driver: clockwise and passenger anticlockwise). 
nique accordingly captures salient relevant features by utilising smartphone sensory data and doors signal (if available) in a connected set-up. This is followed by a suitable classifier and decision criterion to accomplish the identification task prior to the start of a journey. A step by step description of this recognition solution is given in Section IV-A.

The employed features, namely ingress micro-movements, are clearly defined in this paper (see Section IV-B). They are based on the gradient of the heading (yaw) angle with respect to north; the Yaw angle is estimated by fusing the phone's accelerometer, gyroscope and magnetometer data. Contrary to similar existing methods, e.g. [14], [20], we recall that the introduced solution is independent of the vehicle and smartphone position-orientation. It is also tolerant of inaccuracies in the heading angle estimations since it does not use their instantaneous values. For instance, a classifier is directly used on the roll and pitch angles (not yaw) during entry in [14], [20], e.g. from gyroscope; thus their restriction of having the phone in a lower-body pocket. Additionally due to the varying positions-orientations of the phone before and after entry, detecting pedal press or seat belt movements from IMU data (where phone has to be in a certain body side) are not considered here.

The proposed technique also incorporates detecting the start (at time $t_{0}$ ) and end (at time $t_{T}$ ) of an entry action to the vehicle. This can be achieved via detecting a walking and stopping activities from rotated accelerometer observations, i.e. with respect to the Global Coordinates System (GCS). In this paper, we only use the linear accelerations in the GCS (see Section IV-C), which simplifies the step-detection and minimises the required parameters fine-tuning. Additionally, doors signal (if available) from the vehicle OBD/CAN-bus, which reports the status (i.e. open or closed) of all vehicle doors can instead set or rectify the attained $t_{0}$ and $t_{T}$.

Whilst analysing the entry behaviour permits establishing if the phone/user is in the right (driver) or left (P-F) vehicle side, the doors signal can enable differentiating between $\mathrm{D}$ and $\mathrm{P}-\mathrm{RO}$ or $\mathrm{P}-\mathrm{F}$ and $\mathrm{P}-\mathrm{RN}$ in Fig. 1. It is noted that the doors signal alone is insufficient for identification, even if only one user is present, as the vehicle does not know which smartphone/user is approaching-entering. Since obtaining the doors signal by the smartphone involves creating a data link with the vehicle, it can be necessary here only when any of the rear doors (R-PO or R-RN) are opened or more than two users are simultaneously returning to vehicle.

\section{A. Overall System}

Figure 3 depicts the flowchart of the proposed solution; time instant $t_{0}$ denotes the start of the entry and/or end of the walking action. At the arrival of a new accelerometer, magnetometer and gyroscope observations at the current time instant $t$, the system implements:

1) Align the phone axes to GCS; calculate rotation matrix.

2) Detect (steps) walking by fusing phone data; otherwise, repeat the previous action at the next time instant $t+h$ ( $h$ is the time step between two successive IMU sensory measurements).

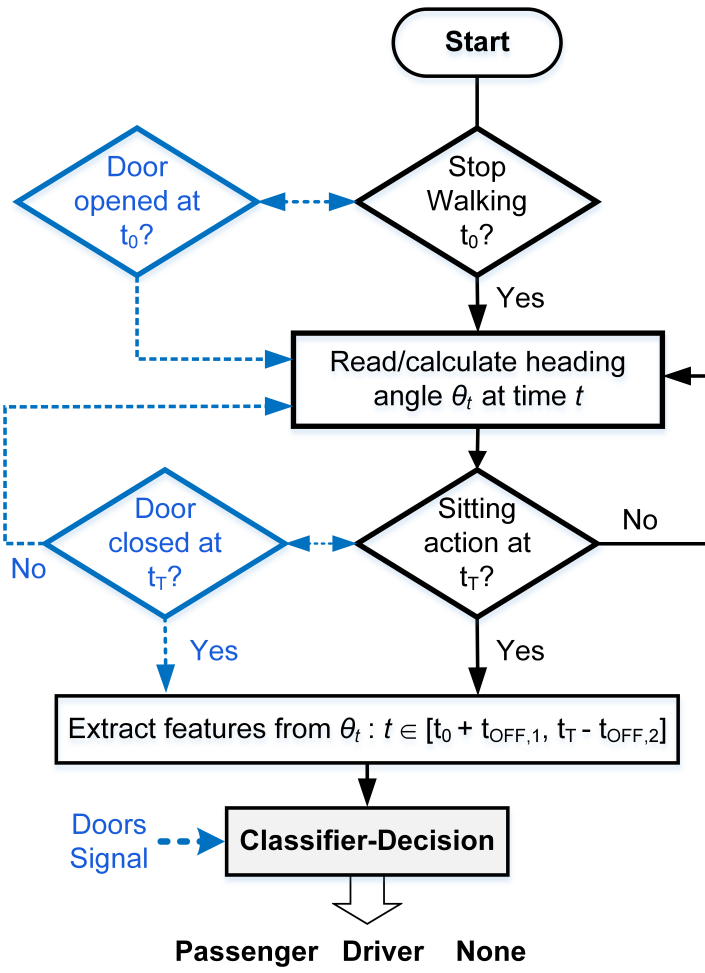

Fig. 3: Overall system flowchart from the onset of entry at $t_{0}$; operations related to the vehicle doors signal are in blue.

3) Detect stop of walking (i.e. start of entry at $t_{0}$ ) via step detection; check for doors open/close events; otherwise restart.

4) Calculate/refine the heading angle $\theta_{t}$ w.r.t. GCS.

5) Detect the sitting event at time $t_{T}$; check doors openclose signal. Otherwise, repeat the previous action.

6) Extract $N$ features $\left\{\psi_{n}\right\}_{n=1}^{N}$ from all calculated $\theta_{t}$ for $t \in\left[t_{0}+t_{\mathrm{OFF}, 1}, t_{T}-t_{\mathrm{OFF}, 2}\right]$ during the entry movements. Offsets $t_{\mathrm{OFF}, 1}$ and $t_{\mathrm{OFF}, 2}$ are introduced to permit adjusting the period of interest in the entry action, e.g. excluding events shortly after entering the vehicle.

7) Apply a classifier to obtain the probability of a user entering from the right (driver) side $p(R \mid \boldsymbol{\psi})$ and left (front passenger) side $p(L \mid \boldsymbol{\psi})$ where $\boldsymbol{\psi}=\left[\psi_{1}, \psi_{2}, \ldots, \psi_{N}\right]^{\prime} ; \boldsymbol{x}^{\prime}$ is the transpose of vector/matrix $\boldsymbol{x}$.

8) Apply a decision criterion; utilise available door signals. The heading (yaw) angles used in the features vector $\boldsymbol{\psi}$ are: i) unwrapped to circumvent erratic changes, e.g. around $\pm 180^{\circ}$, and ii) smoothed with a moving average (filter) to reduce the impact of fast fluctuations. This does not have any visible impact on recognition since the examined features are related to the angle's gradient, rather than its instantaneous values.

Therefore, it is a probabilistic approach that can meet prescribed certainty requirements via a decision criterion. Below, we detail each of the above eight operations, starting with the critical feature extraction, classification and decision aspects.

\section{B. Features, Classification and Decision}

Let $\tilde{\theta}(t)=\partial \theta(t) / \partial t, t \in \mathcal{T}$, be the gradient of the heading angle within $\mathcal{T}=\left[t_{0}+t_{\mathrm{OFF}, 1}, t_{T}-t_{\mathrm{OFF}, 2}\right]$, which is split into $N$ 
nonoverlapping time segments $\mathcal{T}=\mathcal{T}_{1} \cup \mathcal{T}_{2} \ldots \cup \mathcal{T}_{N}$. Whereas,

$$
\psi_{n}=\frac{1}{\mu_{n}} \sum_{t \in \mathcal{T}_{n}} \tilde{\theta}(t), \quad n=1,2, \ldots, N,
$$

and $\mu_{n}$ is the number of differentials $\tilde{\theta}_{t}$ in $\mathcal{T}_{n}$. Hence, the features vector $\boldsymbol{\psi}=\left[\psi_{1}, \psi_{2}, \ldots, \psi_{N}\right]^{\prime}$ of dimensions $N \times 1$ represents the entry turning micro-movements in each time segment $\mathcal{T}_{n}$. It is intuitive to assume that the user undertakes several micro-turning-movements during ingress, e.g. at $20 \%$, $40 \%, 60 \%$ and $80 \%$; each of varying characteristics. They collectively capture the distinctive entry behaviour. It is emphasised that identifying the suitable features is fundamental and several standard classifiers can then be applied [46].

For illustration and since this classification problem has only two classes $\mathcal{C}=\{R, L\}$, the linear logistic regressor/classifier is described here. The resultant probabilities for each class can be expressed by

$$
p(R \mid \boldsymbol{\psi}, \boldsymbol{\beta})=\sigma\left(\boldsymbol{\beta}^{\prime} \boldsymbol{\psi}\right), \quad p(L \mid \boldsymbol{\psi}, \boldsymbol{\beta})=1-p(R \mid \boldsymbol{\psi}, \boldsymbol{\beta}),
$$

where $\boldsymbol{\beta}$ is an $N \times 1$ vector obtained from the training data and $\sigma(z)=1 /\left(1+e^{-z}\right)$ is the logistic function. Maximizing the likelihood from labelled data (i.e. supervised training) can be used to set the optimal values for $\boldsymbol{\beta}$. This can be performed offline from recorded data (i.e. batch learning) or it can be an online learning process, for instance gradient-ascent, Newton's method, etc. We recall that nonlinear logistic classification and other classifiers, such as Support Vector Machines (SVM) can be employed in an analogous way [46].

The dimension of the features vector, i.e. $N$, is a design parameter that should adequately reflect the level of variabilities in the user entry micro-movements. Although the value of $N$ can be fixed in practice (e.g. $N=2$ or $N=5$ ), it can be customised for a particular driver/passenger. For instance, a larger $N$ value can be applied for a user who consistently exhibits drastically changing $\tilde{\theta}(t)$ during entries to vehicle.

Having determined the sought probabilities $p(R \mid \boldsymbol{\psi}, \boldsymbol{\beta})$ and $p(L \mid \boldsymbol{\psi}, \boldsymbol{\beta})$, a decision can be made based on minimising

$$
\hat{\mathcal{C}}=\arg \min _{\mathcal{C}=\{R, L\}} \mathbb{E}\left[c\left(\mathcal{C}, \mathcal{C}^{+}\right) \mid \boldsymbol{\psi}\right]
$$

where $c\left(\mathcal{C}_{i}, \mathcal{C}^{+}\right)$is the cost of an incorrect classification. It can be easily seen that a binary cost function results in a Maximum a Posteriori (MAP) estimate, i.e. the most probable class is chosen without a "none" outcome as in Fig. 3. Alternatively, a threshold criterion can be used, e.g. $p(R \mid \boldsymbol{\psi}, \boldsymbol{\beta}) \geqslant \gamma_{R}$ deems that the user is entering from the vehicle right side. This permits quantifying the certainly level of the identification operation and establishing cases when the system cannot determine, with sufficiently high probability, the user identify.

In scenarios where two or more users (i.e. a driver and passengers) are returning to the vehicle, determining entry from the left or right hand sides can suffice, except for differentiating between a driver and P-RO or front passenger and P-RN in Fig. 1. The doors signal is consequently employed to resolve the in-vehicle position (seat) of the present users, i.e. rear or front. This is handled by linking the smartphone that detected a stop-walking event (as per Section IV-E) closest to the timestamp of a door opening event, see Fig. 7 for an illustration of this basic, yet effective, strategy.

\section{Estimating Rotation Matrix and Heading Angle}

The smartphone's accelerometer, gyroscope and magnetometer observations are with respect to the phone's reference Local Coordinates System (LCS). On the other hand, a global coordinate system has: 1) an $\mathrm{x}$-axis tangential to the ground and is pointing East, 2) y-axis is tangential to the ground and points towards north and 3) z-axis points up and is perpendicular to the ground. A conventional way to perform this coordinate transformation at time $t$ is by the rotation matrix $\mathbf{M}_{t}$ comprised of the following three basic matrices

$$
\begin{gathered}
\mathbf{M}_{x, t}=\left[\begin{array}{ccc}
1 & 0 & 0 \\
0 & \cos \varphi_{t} & -\sin \varphi_{t} \\
0 & \sin \varphi_{t} & \cos \varphi_{t}
\end{array}\right], \mathbf{M}_{y, t}=\left[\begin{array}{ccc}
\cos \phi_{t} & 0 & \sin \phi_{t} \\
0 & 1 & 0 \\
-\sin \phi_{t} & 0 & \cos \phi_{t}
\end{array}\right] \\
\mathbf{M}_{z, t}=\left[\begin{array}{ccc}
\cos \theta_{t} & -\sin \theta_{t} & 0 \\
\sin \theta_{t} & \cos \theta_{t} & 0 \\
0 & 0 & 1
\end{array}\right]
\end{gathered}
$$

where $\varphi_{t}$ (pitch), $\phi_{t}$ (roll) and $\theta_{t}$ (yaw) are the rotation angles about the $x, y$ and $z$ axes, respectively; $\mathbf{M}_{t}=\mathbf{M}_{z, t} \mathbf{M}_{x, t} \mathbf{M}_{y, t}$. An LCS to GCS transformation of a vector, e.g. 3D acceleration vector, is accomplished via $\boldsymbol{a}_{t}^{G C S}=\mathbf{M}_{t} \boldsymbol{a}_{t}^{L C S}$.

By utilising the gravitational accelerations and magnetometer data at time $t$, the rotation matrix can be expressed by, as with the original Android implementation [47],

$$
\mathbf{M}(t)=\left[\begin{array}{lll}
\boldsymbol{e}_{t}^{\prime} & \boldsymbol{n}_{t}^{\prime} & \boldsymbol{u}_{t}^{\prime}
\end{array}\right]^{\prime},
$$

$\boldsymbol{e}_{t}=\boldsymbol{a}_{t}^{\text {grav }} \times \boldsymbol{m}_{t} /\left|\boldsymbol{a}_{t}^{\text {grav }} \times \boldsymbol{m}_{t}\right|, \boldsymbol{n}_{t}=\boldsymbol{e}_{t} \times \boldsymbol{a}_{t}^{\text {grav }} /\left|\boldsymbol{e}_{t} \times \boldsymbol{a}_{t}^{\text {grav }}\right|$ and $\boldsymbol{u}_{t}=\boldsymbol{e}_{t} \times \boldsymbol{n}_{t} /\left|\boldsymbol{e}_{t} \times \boldsymbol{n}_{t}\right|$. Vector $\boldsymbol{a}_{t}^{\text {grav }}$ is the gravitational accelerations from IMU data and $\boldsymbol{m}_{t}$ denotes the magnetometer observation vector; product of two $3 \times 1$ vectors $\boldsymbol{a} \times \boldsymbol{b}=\left(a_{y} b_{z}-a_{z} b_{y}\right) i+\left(a_{z} b_{x}-a_{x} b_{z}\right) j+\left(a_{x} b_{y}-a_{y} b_{x}\right) k$. The Euler angles can be easily attained from $\mathbf{M}_{t}$ using

$$
\begin{gathered}
\varphi_{t}=\sin ^{-1}\left(\mathbf{M}_{t}(3,2)\right), \quad \phi_{t}=\tan ^{-1}\left(-\mathbf{M}_{t}(3,1) / \mathbf{M}_{t}(3,3)\right), \\
\theta_{t}=\tan ^{-1}\left(-\mathbf{M}_{t}(1,2) / \mathbf{M}_{t}(2,2)\right) .
\end{gathered}
$$

It is noted that the accelerometer measures both the gravitational $\mathbf{a}_{t}^{\text {grav }}$ and linear $\mathbf{a}_{t}^{l}$ forces. A low-pass filter extracts the gravitational component for estimating $\mathbf{M}_{t}$ in (5) .

Whilst accelerometers and magnetometers suffer from various sources of errors, the gyroscope has a short response time and is capable of giving accurate measurements of angular speeds, i.e. $\boldsymbol{\omega}_{t}=\left[\begin{array}{lll}\omega_{x, t} & \omega_{y, t} & \omega_{z, t}\end{array}\right]^{\prime}$ around the phone LCS axes. Orientation angles can subsequently be tracked by integrating the gyroscope output. This demands accurate initialisation and the gyroscope observations generally can drift over time leading to erroneous orientations.

Several methods, of varying complexities, fuse results from accelerometer-magnetometer, e.g. $\mathbf{M}_{t}$ in (5), with those from a gyroscope, e.g. a rotation matrix $\mathbf{M}_{t}^{q}$ from $\boldsymbol{\omega}_{t}$ using a quaternion representation. A quaternion $q$ has two parts, a vector $\left[\begin{array}{lll}x & y & z\end{array}\right]^{\prime}$ and a scalar term $\omega$, such that $q=$ $\exp (\varpi(a i+b j+c k) / 2)=\omega+x i+y j+z k, a=\omega_{x, t} / \vartheta_{t}$, $b=\omega_{y, t} / \vartheta_{t}, c=\omega_{z, t} / \vartheta_{t}, \vartheta_{t}=\left\|\boldsymbol{\omega}_{t}\right\|_{2}$ and angle $\varpi=$ $\int_{t}^{t+\delta t} \vartheta_{t} d t$. The left-handed rotation matrix is constructed

$$
\mathbf{M}_{t}^{q}=\left[\begin{array}{ccc}
1-2 y^{2}-2 z^{2} & 2 x y-2 z w & 2 x z+2 y w \\
2 x y+2 z w & 1-2 x^{2}-2 z^{2} & 2 y z-2 x w \\
2 x z-2 y w & 2 y z+2 x w & 1-2 x^{2}-2 y^{2}
\end{array}\right]
$$

and (6) is applied [47]. A simple fusion approach is the complimentary filter: $\theta_{t}=\alpha \theta_{t}^{\text {gyro }}+(1-\alpha) \theta_{t}^{\text {mag,acc }}$ with 
coefficient $\alpha$. Other methods focus on the yaw angle due to its relevance in general navigation tasks, e.g. [24], [28], [48].

As the focus of this paper is not localisation, the complementary filter or the iOS implementation for estimating $\theta_{t}$ can be employed. Heading angle estimation from a phone with arbitrary positions-orientations and low quality inertial data is distinct from navigation systems that use high precision IMUs or IMUs at known (stable) positions, e.g. on a helmet or belt.

\section{Step Detection Using GCS Acceleration Vector}

Similar to calculating the heading (yaw) angle from smartphone data, step detection is critical to PDR and several methods exist [24], [28]. To simplify the detection routine and taper variabilities in the utilised IMU data, in this paper we only use the linear accelerations in the GCS z-axis. This also minimises any fine-tuning of the detector parameters.

After rotating the accelerometer measurement vector at $t$, the z-axis component $a_{z}^{G C S}(t)$ is extracted, i.e. last element in $\mathbf{a}_{t}^{G C S}$. A high-pass filtering (e.g. cut-off frequency $f_{\mathrm{C}}=0.9$ $\mathrm{Hz})$ is first applied to obtain the linear acceleration $a_{z}^{l}(t)$, followed by a low-pass filter to remove high frequency noise (e.g. $f_{\mathrm{C}}=5 \mathrm{~Hz}$ ) yielding $\hat{a}_{z}^{l}(t)$; simple filters can be utilised, such as a moving average. Here, we adopt the three stage thresholding procedure in [28]. Each threshold produces a set of time instants within a predefined time window $\tilde{\mathfrak{T}}=$ $[t-W, t+W]$ around the current observation time $t$, as per

- $T_{\text {Peak }}: \arg \max _{t_{p}} a_{z}^{l}\left(t_{p}\right)$ s.t. $a_{z}^{l}\left(t_{p}\right) \geqslant \gamma_{P}$ and $t_{p} \in \tilde{\mathfrak{T}}$.

- TPeakToValley: ensures that the peak-to-valley difference (before and after a step) is above a certain threshold value $\gamma_{P V}$. This eliminate scenarios where acceleration fluctuations do not vary enough to constitute a step.

- $T_{\text {SlopeGradient }}$ : ensures that the accumulative acceleration slope is negative on the left-side and positive on the front side to eliminate sharp-sudden data fluctuations.

This requires rotated-filtered $\mathrm{z}$-axis accelerations $\hat{a}_{z}^{l}(t)$ for the time window $\tilde{\mathfrak{T}}$. A step is detected with a time lag of $W$; where $W<1 s$ is typical and depends on the IMU data rate.

For a given IMU measurement and time window $\tilde{\mathfrak{T}}$, the detected step(s) time(s), if any, is given by:

$$
t_{\text {Step }}=T_{\text {Peak }} \cap T_{\text {PeakToValley }} \cap T_{\text {SlopeGradient }} .
$$

Figure 4 depicts an example of these times. Thresholds $\gamma_{P}$ and $\gamma_{P V}$ can be set for a common scenario or adaptively modified. The former is often sufficient as in the experiments below.

\section{E. Detect Stop Walking and Sitting Actions}

Let $\mathbb{T}_{\text {Step }}$ be the set of step times $t_{\text {Step }}$ in (8) from the beginning of the ongoing walking action up to the current time instant $t$. An up-to-date running average of step durations, i.e. time gap between two successive steps, is kept and it is denoted by $T_{S \text {,mean }}$. A stop-walking action is detected at time $t_{0}$ if no steps are detected for $\kappa T_{S \text {,mean }}$, for instance $\kappa=2.5$, and a sitting action at time $t_{T}$ is indicated by an additional step-like-activity (with a shorter time window, i.e. smaller $W$ ) measured after the stop action. Several measures can be taken to eliminate short or initial detected steps, e.g. a nominal stop action is preceded by at least $K$ consecutive steps (e.g. $K=$ 8 ). Offset times can be set to $t_{\mathrm{OFF}, 1}=t_{\mathrm{OFF}, 2}=0.5 T_{S \text {,mean }}$.
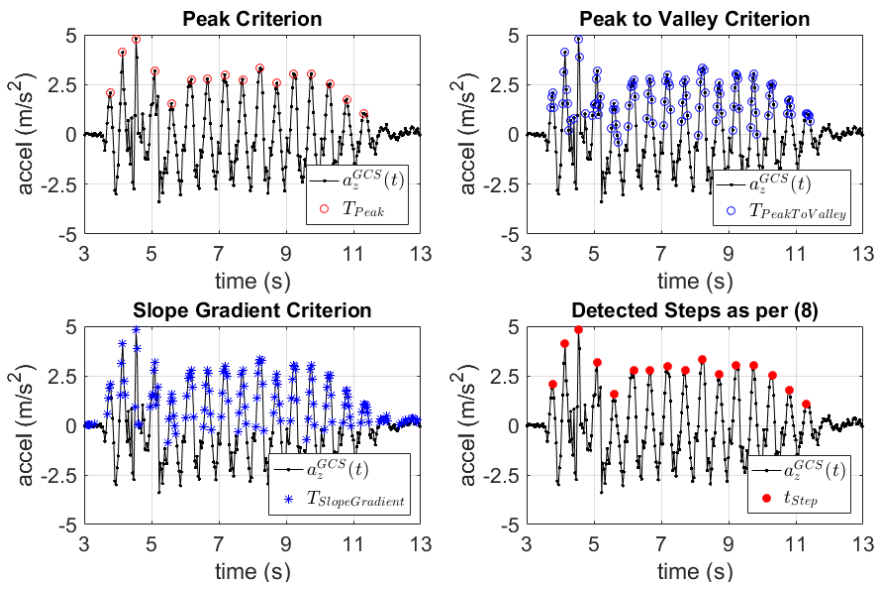

Fig. 4: Step detection with (8) showing $T_{\text {PeakToValley }}$ and $T_{\text {SlopeGradient }}$.

\section{F. Potential Implementation and Final Remarks}

In practice, a user can download and install an Application on his/her smartphone, which implements and seamlessly runs the proposed driver/passenger identification approach as with the last pilot study in the next section. Thus, no specialised/proprietary hardware is needed. The smartphone can receive, in nearly real-time, data from the vehicle, such as the doors signal, and potentially send back (share) the recognition results, for instants via a cloud service, in a connected vehicle enviroment (e.g. with V2X technology [16], [17]). Alternatively, doors signal as well as (refined) identification results can be exchanged after the user's smartphone pairs with the vehicle, e.g. the infotainment system via Bluetooth.

Similar to other smartphone-based identification techniques, a common challenge is determining when the user is near or entering the vehicle, i.e. when to perform the identification operation. Whilst walking-stopping and door signals are used above, the unavailability of the doors signal can lead to: a) collecting-processing smartphone data that does not pertain to an entry action (e.g. non-driving sitting actions in daily life) producing incorrect classifications as well as increasing the power consumption requirements of the identification solution, and b) restrict the recognition capability to left or right side entry which does not permit differentiating between driver and P-RO or P-F and P-RN. Nonetheless, the former limitation can be mitigated by utilising data from other modalities such as phone pairing with vehicle, inaccurate proximity-to-vehicle sensing or localisation, presence of a key-fob, contextual information (e.g. time, calender and learnt pattern of life), etc.

The simplicity of this smartphone-based approach, such that none of its modules apply a complex algorithm, is pivotal to its appeal. Whilst it predominantly relies on capturing salient features from the smartphone IMU data, it presents an effective driver/passenger(s) identification solution as per the results below. Ultimately, it can be applied in conjunction with other detection methods, e.g. those listed in Table II, to improve the overall recognition accuracy.

\section{EXPERIMENTAL RESULTS}

Several pilot experimental studies are conducted in various cars, namely Land Rover and Jaguar XF, XJ and F Pace. The 
total number of assessed approaches-entries is 107 trials/tracks undertaken by seven participants (one female). In these pilot studies, a participant walks to the car from behind, left and right sides of the vehicle. He/she then enters to the designated seat position, namely driver D or front passenger P-F, see Fig. 1. Two phone positions were considered, i.e. in hand and in a front or back trouser pocket; orientation is arbitrary. The split is nearly even between the tested conditions, i.e. approach side, in-car seat and phone position. In each trial, a developed iOS application provides the raw accelerometer, gyroscope and magnetometer data at rates of $30-50 \mathrm{~Hz}$. A CAN-bus analyser reports the doors signal, which is not utilised for identification in these trials. For the step detection and data fusion, the parameters are fixed at $W=0.5 \mathrm{~s}, \gamma_{P}=0.5 \mathrm{~m} / \mathrm{s}^{2}$ and $T_{\text {PeakToValley }}=1.5 \mathrm{~m} / \mathrm{s}^{2}$ and $\alpha=0.95$. Whilst 2-D features vector $\psi \in \mathbb{R}^{2}$, for equal partitions $\left|\mathcal{T}_{1}\right|=\left|\mathcal{T}_{2}\right|$, and equal weights are used, a MAP decision is applied. Thus, the output is either a driver (class $R$ ) or passenger (class $L$ ) as per (2).

Overall, the identification success rate of the introduced approach is approximately $93 \%$ in all trials without using the doors signal. The phone position (hand or pocket) did not have a visible impact on the success rate and majority of incorrect classifications are attributed to erroneous estimations of $t_{0}$, i.e. detecting a stopping-entering event.

Figure 5 shows two examples where a user walks and enters the vehicle; it displays the heading angle estimates and detected steps from phone data. It can be noticed that a driver turns clockwise and front passenger turns anticlockwise during entry. Figure 6 depicts the 2-D features, $N=2$ in (1), capturing the turning behaviour at $50 \%$ and $100 \%$ of the ingress action from all trials; doors signal is employed to correct $t_{0}$ (when relevant). The figure also shows the decision boundary between the two classes (D and P-F) of the logistic regressor in (2). It is attained from maximising the likelihood function via a gradient-ascent optimiser. Figure 6 clearly illustrates that the selected representative features depend on the side of entry, i.e. entering from left (front passenger) or

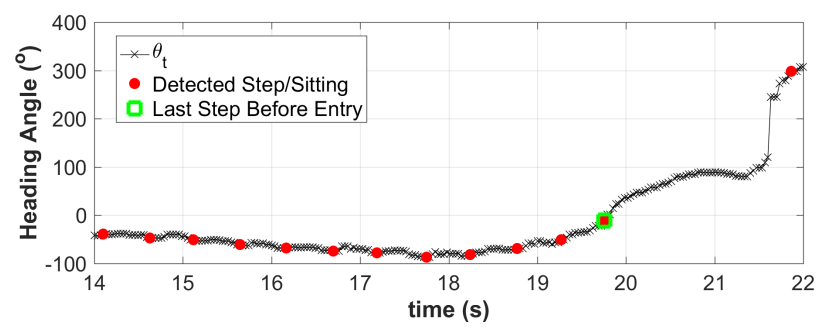

(a) Driver (D) entry from the right side of the forward facing car.

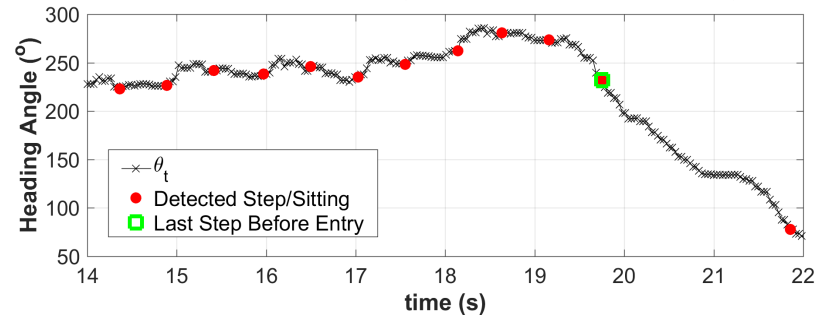

(b) Front Passenger (P-F) entry from left side of the forward facing car.

Fig. 5: Heading angle $\theta_{t}$ and steps for user walking-entering to car.

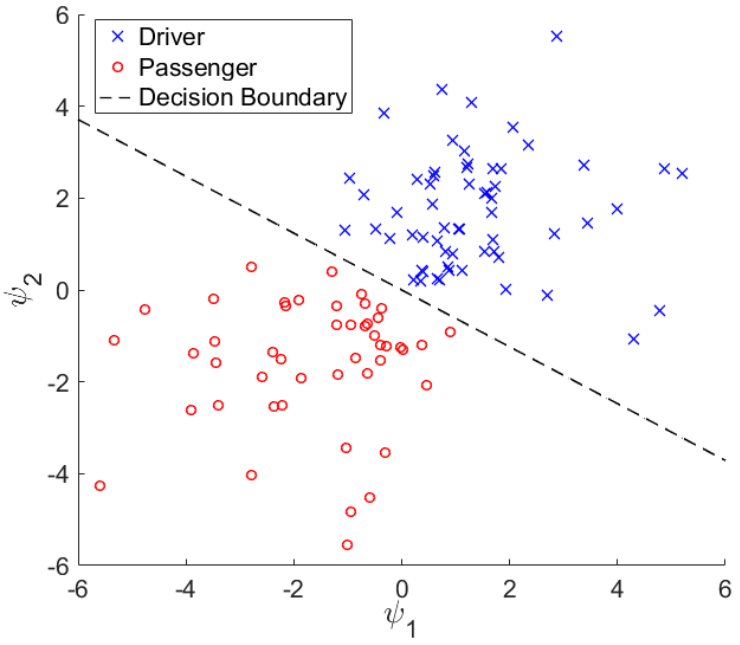

Fig. 6: Considered entry features $(N=2)$ in (1) for a driver and passenger with the decision boundary of the linear logistic regressor.
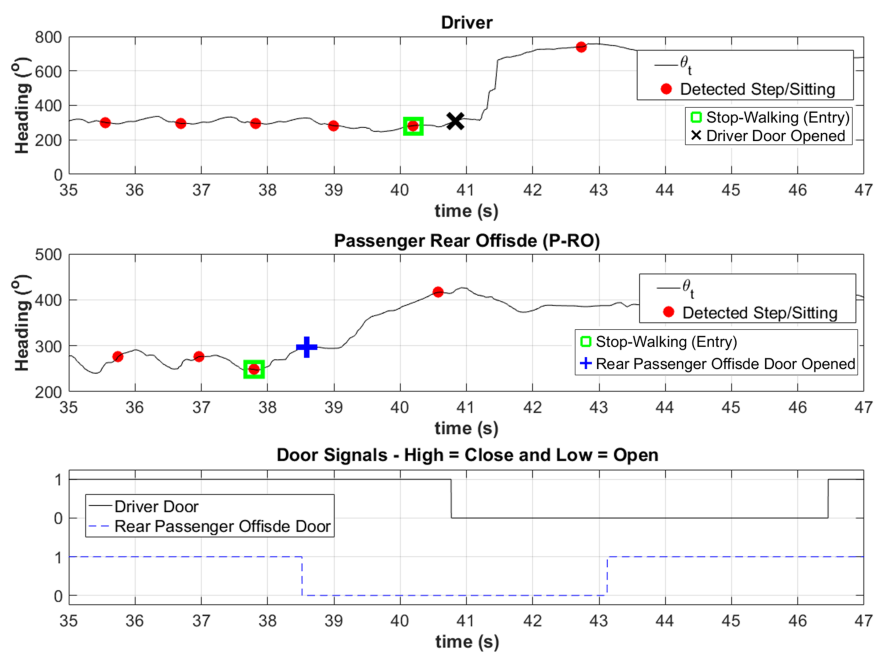

Fig. 7: Heading angle and door signals (high "1" closed and low " 0 " opened) for D and P-RO concurrently approaching-entering car. Green square is the last detected step prior to the corresponding user entering car. The black x-mark and blue plus markers indicate the time instants the D and P-RO doors opened, respectively.

right (driver) sides, and the linear logistic classifier can suffice.

Another pilot study was conducted where: i) a driver first opens the car-boot or R-PO door prior to entering the vehicle, and ii) two or more participants simultaneously approach the car (from behind or left or right) and enter. In both scenarios, the smartphone is either in hand or pocket. An iOS application, which implements all the system components in Section IV, was developed; this incorporates the door signals. The obtained success rates are:

- Driver first opens car boot or P-RO door (to put bag) prior to sitting: $87.5 \%$ (8 trials).

- Driver and P-RO approach-enter car: 100\% (8 trials).

- Four users (D, P-F, P-RN, P-RO) approach-enter vehicle: approximately $90 \%$ (24 trials).

Figure 7 depicts heading angle, steps and doors signal for a driver and R-PO concurrently approaching and entering 
the vehicle, i.e. both enter from the driver (right) side of the vehicle. Thereby, their entry behaviours (direction of the turning angle) is noticeably similar. This figure exhibits how a door signal, i.e. door opening action closest to a detected stopwalking activity, facilitate distinguishing between a driver and P-RO. For example, the time gap between the last detected step prior to entry (marked by the green square at $t=40.1 \mathrm{~s}$ in the top row plot in Fig. 7) and opening the driver door (i.e. the black $\mathrm{x}$-mark at $t=40.9 \mathrm{~s}$ ) is significantly smaller than the time gap between the driver door opening and the P-RO stop-walking event (marked by the green square at $t=37.7 \mathrm{~s}$ in the middle row plot of the figure). Whereas, the P-RO door is opened at $t=38.5 \mathrm{~s}$ (i.e. the blue plus).

In summary, these pilot studies clearly demonstrate the usefulness and effectiveness of the proposed low-complexity smartphone-based identification solution. It is noted that other comparable identification methods, i.e. those which utilise the smartphone IMU data to analysis the user entry behaviour, e.g. [14], [20], produced substantially poor recognition success (less than 50\%) when applied to the collected experimental IMU data, albeit using a more complex classifier such as the SVM and a features vector of dimensions well above the considered $N=2$. This is especially the case for experiments with multiple approaching vehicle users and for a driver who first opens the car boot or another door prior to entering.

\section{CONCLUSIONS}

This paper introduces a simple probabilistic approach for driver/passenger(s) identification using the smartphone and vehicle doors signal (if available). It analyses the user motion during entry and captures salient ingress features. It is shown to deliver a notably high (e.g. exceeding 90\%) in selected pilot studies; even when doors signal is not used. Future work includes extended experimental evaluations in naturalistic settings and devising more principled formulation for associating the doors signal with each present smartphone (i.e. instead of the rule-based approach applied above).

It is emphasised that the strength of the proposed approach is its simplicity. It is expected that it will be employed within a hierarchical system that implements several identification methods at various stages, before and after the start of a new journey, to improve the overall driver/passenger(s) classification robustness and accuracy. This paper serves as impetus to future research into analysing human behaviour, as measured by a smartphone, during and after entering vehicles, to determine the user identify. In a connected set-up, other vehicle signals (e.g. seat belt and pedal-presses) can be employed to aid or guide the identification procedure.

\section{ACKNOWLEDGMENT}

The authors would like to thank Jaguar Land Rover for funding this work under the Centre for Advanced Photonics and Electronics (CAPE) agreement.

\section{REFERENCES}

[1] R. Bishop, Intelligent Vehicle Technology and Trends. Artech House, Inc., 2005

[2] A. Broggi, A. Zelinsky, Ü. Özgüner, and C. Laugier, "Intelligent vehicles," in Springer Handbook of Robotics, 2016, pp. 1627-1656.

[3] K. Bengler, K. Dietmayer, B. Farber, M. Maurer, C. Stiller, and H. Winner, "Three decades of driver assistance systems: Review and future perspectives," IEEE Intelligent Transportation Systems Magazine, vol. 6, no. 4, pp. 6-22, 2014.

[4] A. Paul, R. Chauhan, R. Srivastava, and M. Baruah, "Advanced driver assistance systems," SAE Technical Paper, Tech. Rep., 2016.

[5] R. Karlsson and F. Gustafsson, "The future of automotive localization algorithms: Available, reliable, and scalable localization: Anywhere and anytime," IEEE Signal Processing Mag., vol. 34, pp. 60-69, 2017.

[6] Jaguar Land Rover, "The Worlds First Self-learning Car." Accessed on 15 March 2018 from: https://www.landrover.com/experiences/news/self-driving-car.html.

[7] T. Bando, K. Takenaka, S. Nagasaka, and T. Taniguchi, "Unsupervised drive topic finding from driving behavioral data," in IEEE Intelligent Vehicles Symposium (IV), 2013, pp. 177-182.

[8] J. Wiest, M. Karg, F. Kunz, S. Reuter, U. Kreßel, and K. Dietmayer, "A probabilistic maneuver prediction framework for self-learning vehicles with application to intersections," in IEEE Intelligent Vehicles Symposium (IV), 2015, pp. 349-355.

[9] J. Wahlström, I. Skog, and P. Händel, "Smartphone-based vehicle telematics: A ten-year anniversary," IEEE Transactions on Intelligent Transportation Systems, pp. 1-24, 2017.

[10] G. Li, S. E. Li, B. Cheng, and P. Green, "Estimation of driving style in naturalistic highway traffic using maneuver transition probabilities," Transportation Research: Emerging Technologies, pp. 113-125, 2017.

[11] R. Ansar, P. Sarampakhul, S. Ghosh, N. Mitrovic, M. T. Asif, J. Dauwels, and P. Jaillet, "Evaluation of smart-phone performance for real-time traffic prediction," in IEEE ITSC, 2014, pp. 3010-3015.

[12] P. Hao, K. Boriboonsomsin, G. Wu, and M. Barth, "Probabilistic model for estimating vehicle trajectories using sparse mobile sensor data," in IEEE Inte. Conf. on Intelligent Trans.Sys. (ITSC), 2014, pp. 1363-1368.

[13] D.-W. Koh and H.-B. Kang, "Smartphone-based modeling and detection of aggressiveness reactions in senior drivers," in IEEE Intelligent Vehicles Symposium (IV), 2015, pp. 12-17.

[14] H. Chu, V. Raman, J. Shen, A. Kansal, V. Bahl, and R. R. Choudhury, "I am a smartphone and I know my user is driving," in Int. Conf. on Communication Systems and Networks. IEEE, 2014, pp. 1-8.

[15] R. Coppola and M. Morisio, "Connected car: technologies, issues, future trends," ACM Computing Surveys (CSUR), vol. 49, no. 3, p. 46, 2016.

[16] S. Schwarz, T. Philosof, and M. Rupp, "Signal processing challenges in cellular-assisted vehicular communications: Efforts and developments within 3GPP LTE and beyond," IEEE Signal Processing Magazine, vol. 34, pp. 47-59, 2017.

[17] Z. MacHardy, A. Khan, K. Obana, and S. Iwashina, "V2x access technologies: Regulation, research, and remaining challenges," IEEE Communications Surveys \& Tutorials, 2018.

[18] A. Francillon, B. Danev, and S. Capkun, "Relay attacks on passive keyless entry and start systems in modern cars," in The 18th Annual Network And Distributed System Security Symposium. The Internet Society. Citeseer, 2011

[19] F. D. Garcia, D. Oswald, T. Kasper, and P. Pavlidès, "Lock it and still lose it-on the (in) security of automotive remote keyless entry systems." in USENIX Security Symposium, 2016.

[20] C. Bo, X. Jian, T. Jung, J. Han, X.-Y. Li, X. Mao, and Y. Wang, "Detecting drivers smartphone usage via nonintrusively sensing driving dynamics," IEEE Internet of Things Journal, vol. 4, no. 2, pp. 340-350, 2017.

[21] J. Zaldivar, C. T. Calafate, J. C. Cano, and P. Manzoni, "Providing accident detection in vehicular networks through OBD-II devices and android-based smartphones," in IEEE 36th Conference on Local Computer Networks (LCN), 2011, pp. 813-819.

[22] P. D. Groves, Principles of GNSS, inertial, and multisensor integrated navigation systems. Artech house, 2013.

[23] A. J. Haug, Bayesian Estimation and Tracking: A Practical Guide. John Wiley \& Sons, 2012.

[24] P. Davidson and R. Piche, "A survey of selected indoor positioning methods for smartphones," IEEE Comm. Surveys \& Tutorials, vol. 19 , pp. $1347-1370,2016$

[25] S. Tarkoma, M. Siekkinen, E. Lagerspetz, and Y. Xiao, Smartphone energy consumption: modeling and optimization. Cambridge University Press, 2014. 
[26] S. Hu, L. Su, S. Li, S. Wang, C. Pan, S. Gu, M. T. Al Amin, H. Liu, S. Nath, R. R. Choudhury et al., "Experiences with eNav: A lowpower vehicular navigation system," in Proceedings of the 2015 ACM International Joint Conference on Pervasive and Ubiquitous Computing. ACM, 2015, pp. 433-444.

[27] R. Faragher and R. Harle, "Location fingerprinting with Bluetooth low energy beacons," IEEE Journal on Selected Areas in Communications, vol. 33, no. 11, pp. 2418-2428, 2015.

[28] W. Kang and Y. Han, "SmartPDR: Smartphone-based pedestrian dead reckoning for indoor localization," IEEE Sensors Journal, vol. 15, no. 5, pp. 2906-2916, 2015.

[29] V. Pasku, A. De Angelis, G. De Angelis, D. D. Arumugam, M. Dionigi, P. Carbone, A. Moschitta, and D. S. Ricketts, "Magnetic field based positioning systems," IEEE Communications Surveys \& Tutorials, 2017.

[30] B. I. Ahmad, T. Ardeshiri, S. J. Godsill, P. M. Langdon, and T. Popham, "Modelling received signal strength from on-vehicle BLE beacons using skewed distributions: A preliminary study," in Proc. of the 20th International Conference on Information Fusion (Fusion '17), 2017, pp. $1-7$.

[31] A. Alarifi, A. Al-Salman, M. Alsaleh, A. Alnafessah, S. Al-Hadhrami, M. A. Al-Ammar, and H. S. Al-Khalifa, "Ultra wideband indoor positioning technologies: Analysis and recent advances," Sensors, vol. 16, no. 5 , p. 707,2016

[32] L. Cheng and T. Qiao, "Localization in the parking lot by parked-vehicle assistance," IEEE Transactions on Intelligent Transportation Systems, vol. 17, no. 12, pp. 3629-3634, 2016.

[33] M. Van Ly, S. Martin, and M. M. Trivedi, "Driver classification and driving style recognition using inertial sensors," in IEEE Intelligent Vehicles Symposium (IV), 2013, pp. 1040-1045.

[34] C. Miyajima and K. Takeda, "Driver-behavior modeling using on-road driving data: A new application for behavior signal processing," IEEE Signal Processing Magazine, vol. 33, no. 6, pp. 14-21, 2016.

[35] L. Liu, C. Karatas, H. Li, S. Tan, M. Gruteser, J. Yang, Y. Chen, and R. P. Martin, "Toward detection of unsafe driving with wearables," in Proceedings of the 2015 workshop on Wearable Systems and Applications. ACM, 2015, pp. 27-32.

[36] Y. Wang, Y. J. Chen, J. Yang, M. Gruteser, R. P. Martin, H. Liu, L. Liu, and C. Karatas, "Determining driver phone use by exploiting smartphone integrated sensors," IEEE Transactions on Mobile Computing, vol. 15, no. 8, pp. 1965-1981, 2016.

[37] Z. He, J. Cao, X. Liu, and S. Tang, "Who sits where? infrastructure-free in-vehicle cooperative positioning via smartphones," Sensors, vol. 14, no. 7 , pp. $11605-11628,2014$.

[38] J. Wahlström, I. Skog, P. Händel, and A. Nehorai, "IMU-based smartphone-to-vehicle positioning," IEEE Transactions on Intelligent Vehicles, vol. 1, no. 2, pp. 139-147, 2016.

[39] J. Yang, S. Sidhom, G. Chandrasekaran, T. Vu, H. Liu, N. Cecan, Y. Chen, M. Gruteser, and R. P. Martin, "Sensing driver phone use with acoustic ranging through car speakers," IEEE Transactions on Mobile Computing, vol. 11, no. 9, pp. 1426-1440, 2012.

[40] M. Feld, T. Schwartz, and C. Müller, "This is me: using ambient voice patterns for in-car positioning," in International Joint Conference on Ambient Intelligence. Springer, 2010, pp. 290-294.

[41] R. Cooper, F. Lane, S. Gujral, P. Jacobs, R. Chandhok, L. Ben-Zur, and S. Sharma, "System and method for configuring an interior of a vehicle based on preferences provided with multiple mobile computing devices within the vehicle," Jun. 7 2016, US Patent 9,358,940.

[42] A. Montanari, S. Nawaz, C. Mascolo, and K. Sailer, "A study of Bluetooth low energy performance for human proximity detection in the workplace," IEEE Pervasive Computing, 2017.

[43] M. M. Scheunemann, K. Dautenhahn, M. Salem, and B. Robins, "Utilizing Bluetooth low energy to recognize proximity, touch and humans," in IEEE Int. Symp. on Robot and Human Inter. Comm., 2016, pp. 362-367.

[44] N. Wahlström and F. Gustafsson, "Magnetometer modeling and validation for tracking metallic targets," IEEE Transactions on Signal Processing, vol. 62, no. 3, pp. 545-556, 2014.

[45] K. Masuda, K. Sasaki, K. Obata, H. Yamazaki, and T. Nishimoto, "Carseat-occupant sensing device," Dec. 9 2003, US Patent 6,661,341.

[46] C. Bishop, "Pattern recognition and machine learning," Springer, $N Y$, 2007.

[47] Android, Accessed on 29/03/2017: https://developerandroid.com/guide/topics/_sensors/sensors_motion.html.

[48] J. Favre, B. Jolles, O. Siegrist, and K. Aminian, "Quaternion-based fusion of gyroscopes and accelerometers to improve 3D angle measurement," Electronics Letters, vol. 42, no. 11, pp. 612-614, 2006.
Bashar I. Ahmad (M '11) received the B.Eng. (Hons.) in Electronic Engineering and Ph.D. degrees from the University of Westminster, U.K. in 2007 and 2011, respectively. He is currently a Senior Research Associate in the Signal Processing and Communications (SigProC) Laboratory, Engineering Department, Cambridge University, U.K. and a fellow of Wolfson College, Cambridge. Prior to joining Cambridge University, he was a postdoctoral researcher in the Signal Processing and Communications group at Imperial College London, U.K. His research interests include theory and applications of statistical signal processing and machine learning, such as in intelligent vehicles, sensing systems, automation, data analytics, defense, behavior modelling, wireless communications, human computer interaction and others.

Patrick M. Langdon is a Principal Research Associate for the Engineering Department, Cambridge University, U.K., and a lead researcher in inclusive design within the Engineering Design Centre. He received the undergraduate and Ph.D. degrees from the University of Sheffield, U.K. He has originated numerous collaborative research projects in design for inclusion, automotive, and HMI since joining the department in 1997. He has published extensively in various formats, as author, issues editor and co-author. Currently, he is the PI or Co-I of several industrial automotive projects, and a Co-PI of a 4 year EPSRC project on Autonomous Driving.

Jiaming Liang received a B.Eng. in Electronic and Electrical Engineering from the University of Birmingham, U.K., and a B.Eng. in Communication Engineering from Huazhong University of Science and Technology. He joined the SigProC Laboratory, Engineering Department, Cambridge University, U.K., as a Ph.D. student after obtaining an M.Phil. degree in Advanced Computer Science from the University of Cambridge. His research interests include Bayesian object tracking, dynamic models and Monte Carlo methods.

Simon J. Godsill (M '95) received the B.A. and Ph.D. degrees from the University of Cambridge, U.K., in 1989 and 1994, respectively. He is Professor of statistical signal processing in the Engineering Department at Cambridge University, Cambridge, U.K., and a Professorial Fellow and tutor at Corpus Christi College, Cambridge. He coordinates an active research group in Signal Inference and Its Applications within the Signal Processing and Communications Laboratory at Cambridge, specializing in Bayesian computational methodology, multiple object tracking, audio and music processing, and financial time series modeling. A particular methodological theme over recent years has been the development of novel techniques for optimal Bayesian filtering and smoothing, using sequential Monte Carlo or particle filtering methods. He has published extensively in journals, books, and international conference proceedings. He is a Director of CEDAR Audio Ltd. (which has received a technical Oscar for its audio processing work), and Input Dynamics Ltd., both companies which use his research work in the audio area. Prof. Godsill was Technical Chair of the IEEE NSSPW Workshop in 2006 on sequential and nonlinear filtering methods, was Technical Chair for Fusion 2010 in Edinburgh, U.K., and has been on the conference panel for numerous other conferences and workshops. He has served as Associate Editor for the IEEE TRANSACTIONS ON SIGNAL PROCESSING and the journal Bayesian Analysis. He was Theme Leader in Tracking and Reasoning over Time for the U.K.s Data and Information Fusion Defence Technology Centre (DIF-DTC) and Principal Investigator on grants funded by the EU, EPSRC, QinetiQ, General Dynamics, MOD, Microsoft UK, Citibank, and Mastercard. In 2009-2010, he was co-organiser of an 18-month research program in sequential Monte Carlo methods at the SAMSI Institute, Rayleigh, NC.

Mauricio Delgado received a B.Sc. from the University of Freiburg, Germany in 2012 and a M.Sc. with Honors in Software Engineering from the Technical University of Munich in 2014, having spent a year at MIT, USA, researching machine learning in the field of human factors. He joined Jaguar Land Rover in Coventry, U.K. in 2015 as a Machine Learning Engineer.

Thomas Popham received an M.Eng. in Mechanical Engineering and Ph.D. in Computer Science from the University of Warwick, U.K. in 2006 and 2010, respectively. He then joined Jaguar Land Rover, Coventry, U.K., and is currently the Technical Specialist for Machine Learning and Delivery Manager for Programmable Car and Platforms. 